\title{
Review of: "Battery-free, wireless soft sensors for continuous multi-site measurements of pressure and temperature from patients at risk for pressure injuries"
}

\section{Alexander Schmitz}

Potential competing interests: The author(s) declared that no potential competing interests exist.

I found this paper to have a strong contribution and a clear presentation.

The motivation ("pressure injuries") is clear and substantiated with numbers.

Related work was informative, and the differences to the current work were clear.

The wireless power and data transfer, the sensor structure, and the sensing characteristics are interesting.

I have only minor criticisms:

I doubt that the sensor has no hysteresis. I rather expect the hysteresis to be low.

Some details about the force sensor structure were not clear to me. 